\title{
Fatigue-like effects in the cooperative mechanism revealed with side-by-side reversible figures
}

\author{
CRISTEN B. CORROZI, GERALD M. LONG, and THOMAS C. TOPPINO \\ Villanova University, Villanova, Pennsylvania
}

\begin{abstract}
Subjects viewed two rotating Necker cubes presented simultaneously on opposite sides of a fixation point. During successive viewing periods within the experimental session, the subjects indicated whether the two test figures rotated in unison or in different directions. Within each viewing period, there was a significant decrease in the duration for which the cubes were perceived as rotating together. The possibility of fatigue-like properties within the hypothesized global cooperative mechanism are discussed.
\end{abstract}

The multistable nature of reversible figures such as the Necker cube, Boring's wife/mother-in-law, or the ambiguous triangles allows the viewer to perceive two or more distinct perceptual configurations from an unchanging stimulus. Over the years, numerous researchers have attempted to uncover the perceptual processes responsible for such phenomenal reversals; in general, the empirical work has been driven by two distinct theoretical models. On the one hand, several investigators have claimed that lower level neural processes such as fatigue and recovery of cortical structures play dominant roles in the pattern of reversals (e.g., Babich \& Standing, 1981; Carlson, 1953; von Grünau, Wiggin, \& Reed, 1984). On the other hand, several investigators have claimed that higher level neural processes such as perceptual learning, contextual set, and decision-making play critical roles (e.g., Bugelski \& Alampay, 1961; Reisberg \& O’Shaughnessey, 1984; Rock \& Mitchener, 1992). As described elsewhere (Long, Toppino, \& Mondin, 1992; Toppino \& Long, 1987), extensive empirical evidence has been found to support each of these general theoretical positions. Consequently, researchers have recently favored a more integrative model in which both low-level neural processes and high-level cognitive processes are involved in the perception of reversible figures (e.g., Hochberg \& Peterson, 1987; Long et al., 1992; Palmer \& Bucher, 1981).

Consistent with a multiprocess model of reversible figures has been the frequent use of these figures as research tools. Over the years, investigators have employed reversible figures in the study of such processes as perceptual set (Fisher, 1967), attentional effects (Hochberg \& Peterson, 1987; Peterson \& Gibson, 1991), cognitive influences on figure-ground segregation (Peterson, Harvey, \& Weidenbacher, 1991), and others. Of direct relevance to

The results reported here were taken from one of the experiments in a thesis submitted by the first author for partial completion of the requirements for the Master of Science degree at Villanova University. Reprint requests should be sent to G. M. Long, Department of Psychology, Villanova University, Villanova, PA 19085. the present work are the studies that have used reversible figures in the investigation of global and local processing effects within the perceptual system.

In their (1981) work with ambiguous equilateral triangles, Palmer and Bucher found that global grouping processes can influence how the individual element is perceived. Analogous research was undertaken by Ramachandran and Anstis (1983, 1985), who used bistable apparent motions displays. In these displays, "two spots were flashed simultaneously on diagonally opposite corners of a square and then switched off and replaced by two spots appearing on the remaining corners" ( $\mathrm{Ra}$ machandran \& Anstis, 1983, p. 529). Subjects perceived the flashes as either vertical or horizontal apparent movements of the dots, and the direction of movement could spontaneously reverse with extended viewing. When Ramachandran and Anstis presented their subjects with several identical displays simultaneously throughout the visual field, the subjects reported that the individual dots in the displays seemed to "lock in" so that all the displays exhibited motion in the same direction and flipped at the same time. Ramachandran and Anstis proposed that the operation of a global cooperative mechanism caused all the local displays to remain "in sync."

In the present experiment, we focused on the processes that underlie the cooperative mechanism. Specifically, does the hypothesized cooperative mechanism itself vary over a viewing period in a manner suggestive of fatiguing neural processes? Previous researchers have reported somewhat conflicting results pertaining to the simultaneous perceptions of multiple reversible figures. Long and Toppino (1981), Long, Toppino, and Kostenbauder (1983), and Toppino and Long (1987) reported that reversible figures presented to the two visual fields can reverse separately. In contrast, Ramachandran and Anstis (1985) found that simultaneous ambiguous displays reverse in sync. $\mathrm{Al}-$ though there were several differences across these studies, one possible explanation for these apparent perceptual differences may be that the cooperative mechanism itself is subject to fatigue. If the proposed mechanism were to fa- 
tigue with extended viewing, one would expect conflicting reports due to differences in the duration of the viewing period.

\section{METHOD}

\section{Subjects}

Forty Villanova University students were recruited on a volunteer basis for participation in the study. Each individual received either $30 \mathrm{~min}$ of credit toward the fulfillment of a research requirement for a general psychology course, or a cash payment of $\$ 3.50$ for their participation.

\section{Apparatus}

The apparatus was similar to that previously used by Long et al. (1983). The subjects were seated $1 \mathrm{~m}$ from a screen with a $125 \times$ $265 \mathrm{~mm}$ display window, which consisted of a translucent pane of glass covered by a piece of medium-weight paper that served as a projection screen. Two shadows from a single rotating Necker cube $(44 \mathrm{~mm} / \mathrm{side})$ were projected onto the screen by using two 150 -W light bulbs positioned $95 \mathrm{~cm}$ apart. The luminance on the screen was $3.4 \mathrm{~cd} / \mathrm{m}^{2}$, and the shadows were projected so that their centers were $40 \mathrm{~mm}$ from the centrally located fixation point. The cubes rotated at a constant rate of $10 \mathrm{rpm}$. The subjects placed their fingers on a standard telegraph key that was connected to a Kwik-Set Lab-Chron (No. 1407) electric timer. Depending upon the instruction condition to which they had been assigned, the subjects reported when they perceived the cubes to be rotating in the same (or opposite) direction by pressing and holding the telegraph key. The duration of each response was automatically recorded by the timer.

\section{Procedure}

The subjects were randomly assigned to one of two instruction conditions. Twenty subjects were instructed to report the reversals of the two cubes when they were perceived as rotating in the "same" direction, and 20 subjects were instructed to report when they were perceived as rotating in "opposite" directions. Each experimental session consisted of three 3-min trials separated by 3-min rest periods. During each trial, the subjects viewed two side-by-side ambiguous test figures and depressed and held a telegraph key whenever they perceived the two figures to be rotating in the same (or opposite) direction. In each of the six 30 -sec intervals comprising the 3-min viewing period, the experimenter recorded the duration for which the telegraph key had been depressed. This procedure was repeated for each of the three viewing periods. A 3-min rest period separated each viewing period. Prior to starting the experiment, the subjects were given practice trials in order to familiarize themselves with the procedures and to ensure that they were comfortable with reporting the perceptual alternatives for the rotating Necker cube.

\section{RESULTS AND DISCUSSION}

In this experiment, we focused on the possibility of fatigue within the proposed cooperative mechanism. It was hypothesized that extended viewing of side-by-side figures would lead to fatigue-like effects similar to those reported when viewing a single reversible figure (e.g., Long et al., 1983). If fatigue is found to affect the cooperative mechanism as well, this could help to explain why previous researchers (e.g., Ramachandran \& Anstis, 1983; Toppino \& Long, 1987) found apparently differing results in studies using side-by-side reversible figures.

There were two response groups in this experiment: 20 subjects reported when the two cubes were rotating in the same directions, and 20 subjects reported when the cubes were rotating in opposite directions. For each 30 -sec interval from this latter group of subjects, the data were subtracted from the maximum viewing time of $30 \mathrm{sec}$ in order to provide response data that reflected the duration of time that each subject perceived the figures as rotating in the same direction. Logarithmic transformations were also performed on the raw scores in order to normalize the data. However, this manipulation proved to have no effect on the outcome of the analysis. For the sake of simplicity, the analysis reported here is based on the original data.

The data were analyzed using a 3 (viewing periods) $\times$ 6 (successive 30-sec intervals in 3-min viewing periods) within-subjects analysis of variance. No significant differences were found across the three viewing periods $[F(2,94)=1.22, p>.05]$ and there was no significant interaction between the main effects of viewing period and viewing interval $(F<1.0)$. However, significant differences in the duration measures were found across the successive 30 -sec intervals that comprised the viewing periods $[F(5,235)=5.10, p<.001]$. The mean durations of each 30-sec interval for which the observers reported cooperativity of the two cubes were $20.18,19.35$, $17.95,17.55,18.22$, and $17.98 \mathrm{sec}$, respectively. ${ }^{1} \mathrm{~A}$ series of planned pairwise comparisons among these mean durations revealed significant differences between Interval 1 and Intervals $3,4,5$, and $6[F(1,235)>9.54$, $p<.05$ ], and between Interval 2 and Intervals 3, 4, and $6[F(1,235)>4.64, p<.05]$. Hence, the subjects reported that the ambiguous test figures were rotating in the same direction for longer durations in the early intervals than in the later intervals. (Note that pilot work indicated that if shorter viewing intervals of 5-10 sec are used, nearly complete cooperativity is reported in the early portions of each 3-min trial.)

It might be suggested that the increased independence of reversals across the successive viewing periods involves perceptual learning, with the subjects becoming more facile at separating their perceptions. However, if learning did occur within the 3-min viewing periods, certain predictions concerning the reversal rate across each of the three trials would appear to be reasonable. Specifically, one would also predict a general decrease in cooperativity across successive trials because of savings from one trial to the next. This was clearly not the case, and the similarity of the three successive trials is most consistent with a neural fatigue model and, specifically, with the hypothesized role of fatigue within the cooperative mechanism itself.

An alternative explanation that is not so easily dismissed involves the possibility that the perception of the two rotating Necker cubes is determined by largely independent mechanisms but that each viewing interval begins with both mechanisms favoring the same direction of motion because of stronger cues for that percept. Then, as fatigue of each mechanism builds during the viewing interval, the dominant percept will eventually give way to the alternative (and then eventually back to the original) but at slightly different rates, because the mechanisms cannot be expected to have identical fatigue and recovery rates. In other words, such a model would interpret the present findings as resulting from similar but independent mechanisms that have the same "starting point." The 
present work does not permit a test of this alternative, but its likelihood as the basis for the group reversals with either the Ramachandran and Anstis bistable motion displays or the Palmer and Bucher ambiguous-triangle displays would appear quite forced. It does not appear likely that the individual neural mechanisms responding to local elements of these multiple-element displays would remain in precise synchrony for even brief viewing periods. ${ }^{2}$

\section{GENERAL CONCLUSIONS}

Over the past decade, researchers using a variety of procedures have repeatedly found evidence supporting the hypothesis that neural processes do not work in isolation; local processes work together (or are organized by global influences) to provide us with a wholistic view of the world (see Kimchi, 1992, for a recent review). In the present paradigm with multiple reversible figures, it seems that, although we are capable of perceiving multiple stimuli independently (e.g., Toppino \& Long, 1987; von Grünau et al., 1984), it is a more natural reaction to group the separate percepts together and process the information in a more global fashion. That is, a global cooperative mechanism overlays more localized neural processing, and this mechanism enables us to coordinate our perceptions and to process information in an organized, wholistic manner.

Concerning the basis for such global processing effects, it was hypothesized that the cooperative mechanism may be subject to fatigue effects if it depends critically on cortical channel activity (e.g., Palmer $\&$ Bucher, 1981). We found that extended viewing of multiple reversible figures led to fatigue-like effects, in which the subjects reported increased independence of the individual elements across the viewing period. The fact that the two test figures were initially perceived as reversing together and then began to separate with extended viewing, and that this same pattern was repeated in successive viewing periods supports the proposed concepts of a global cooperative mechanism that is itself subject to fatigue-like effects.

\section{REFERENCES}

Attneave, F. (1968). Triangles as ambiguous figures. American Journal of Psychology, 81, 447-453.

Babich, S., \& STANDING, L. (1981). Satiation effects with reversible figures. Perceptual \& Motor Skills, 52, 203-210.

BUgelski, B. R., \& Alampay, D. A. (1961). The role of frequency in developing perceptual sets. Canadian Journal of Psychology, 15, 205-211.

Carlson, V. R. (1953). Satiation in a reversible perspective figure. Journal of Experimental Psychology, 43, 442-448.

FisHER, G. H. (1967). Preparation of ambiguous stimulus materials. Perception \& Psychophysics, 2, 421-422.

Hochberg, J., \& Peterson, M. A. (1987). Piecemeal organization and cognitive components in object perception: Perceptually coupled responses to moving objects. Journal of Experimental Psychology: General, 116, 370-380.
KIмchI, R. (1992). Primacy of wholistic processing and global/local paradigm: A critical review. Psychological Bulletin, 112, 24-38.

LoNG, G. M., \& TopPINo, T. C. (1981). Multiple representations of the same reversible figure: Implications for cognitive decisional interpretations. Perception, 10, 231-234.

Long, G. M., Toppino, T. C., \& Kostenbauder, J. F. (1983). As the cube turns: Evidence for two processes in the perception of a dynamic reversible figure. Perception \& Psychophysics, 34, 29-38.

Long, G. M., Toppino, T. C., \& Mondin, G. W. (1992). Prime time: Fatigue and set effects in the perception of reversible figures. Perception \& Psychophysics, 52, 609-616.

Palmer, S. E., \& BUCher, N. M. (1981). Configural effects in perceived pointing of ambiguous triangles. Journal of Experimental Psychology: Human Perception \& Performance, 7, 88-114.

Peterson, M. A., \& Gibson, B. S. (1991). Directional spatial attention within an object: Altering the functional equivalence of shape descriptions. Journal of Experimental Psychology: Human Perception \& Performance, 17, 170-182.

Peterson, M. A., Harvey, E. M., \& Weidenbacher, H. J. (1991). Shape recognition contributions to figure-ground reversal: Which route counts? Journal of Experimental Psychology: Human Perception \& Performance, 17, 1075-1089.

RamaChandran, V. S., \& ANSTis, S. M. (1983). Extrapolation of motion path in human visual perception. Vision Research, 23, 83-85.

Ramachandran, V. S., \& ANSTis, S. M. (1985). Perceptual organization in multistable apparent motion. Perception, 14, 135-143.

Reisberg, D., \& O'Shaughnessey, M. (1984). Diverting subjects' concentration slows figural reversal. Perception, 13, 461-468.

Rock, I., \& MitcheNer, K. (1992). Further evidence of failure of reversal of ambiguous figures by uninformed subjects. Perception, 21, $39-45$

Toppino, T. C., \& LonG, G. M. (1987). Selective adaptation with reversible figures: Don't change that channel. Perception \& Psychophysics, 42, 37-48.

von Grünau, M. W., Wiggin, S., \& ReEd, M. (1984). The local character of perspective organization. Perception \& Psychophysics, 35, 319-324.

\section{NOTES}

1. Although it might at first appear that the total range of durations obtained in this work was rather limited, it should be remembered that the total range of possible duration scores extended from $30 \mathrm{sec}$ for complete cooperativity of the two cubes during each 30 -sec interval to $15 \mathrm{sec}$ for complete independence of the cubes during an interval.

2 . We realize that this argument depends on the assumption that the apparent cooperativity obtained with the present figures is due to the same type of neural processes that underlie the cooperativity obtained by these other authors with very different ambiguous stimuli. Additional work is needed to determine the merit of this assumption. Nonetheless, our strong impression with the multiple-triangle display first introduced by Attneave (1968) is that, with extended viewing, the global organization that is initially so strong also begins to break down.

(Manuscript received June 1, 1993.) 